\title{
The van Hove Singularity and Two-Dimensional Superconductivity. Exact Analytical Results
}

\author{
R. SzCZĘŚNIAK ${ }^{a}$ AND S. GRABiŃSki ${ }^{b}$ \\ ${ }^{a}$ Department of Theoretical Physics, Institute of Physics \\ University of Silesia, Uniwersytecka 4, 40-007 Katowice, Poland \\ ${ }^{b}$ Silesian Academy of Medicine, Institute of Biophysics \\ 40-007 Katowice, Poland
}

(Received October 8, 2001; revised version March 11, 2002)

\begin{abstract}
We investigate a weak-coupling approach to superconductivity in the density of states that develops in the two-dimensional lattice with the van Hove singularity located at the Fermi level. Exact analytical expressions for the superconducting order parameter at zero temperature $(\Delta(0))$ and for discontinuity in the specific heat at critical temperature $(\Delta C)$ were derived. We show that the presence of the logarithmic singularity hardly affects the ratio $2 \Delta(0) / k T_{c}$, whereas the temperature dependence of $\Delta C$ can pronouncedly deviate from the standard BCS result.
\end{abstract}

PACS numbers: $74.25 . \mathrm{Kc}$

\section{Introduction}

Since 1986 [1] a lot of experimental and theoretical work has been carried out in order to understand the mechanism of high-temperature superconductivity. Despite serious efforts the basic questions related to this phenomenon remain open problems. The qualitative and quantitative differences between the systems under consideration and conventional superconductors show up both in normal and superconducting state. These differences are mainly attributed to the presence of strong Coulomb interaction, phonon-free contribution to pairing correlations [2] and the two-dimensional character of high-temperature superconductivity, which develops in copper-oxygen planes. Although, it is rather widely believed 
that the high- $T_{c}$ superconductors cannot be properly described within the usual BCS theory, it seems interesting to investigate which properties of these materials require a completely new approach and which can be explained within a modified weak-coupling theory. In particular, it has been shown that the van Hove singularity can result in an important lowering of the isotope shift exponent [3] and can lead to a positive curvature of the upper critical field [4]. Therefore, this particular feature of the two-dimensional density of states can lead to qualitative differences with respect to results obtained within the standard BCS approach. In order to obtain a superconducting phase transition one needs a finite coupling between the $2 \mathrm{D}$ planes. However, we assume that this coupling is small enough to neglect its contribution to the gap equation.

\section{Results and discussion}

In the present paper we discuss the impact of the van Hove singularity on the basic superconducting properties. We obtain accurate analytical expressions for the critical temperature $\left(T_{c}\right)$, the order parameter at zero temperature $(\Delta(0))$, the ratio $2 \Delta(0) / k T_{c}$, temperature dependence of the order parameter and the discontinuity in the specific heat that occurs at the superconducting phase transition. The problems considered in the present paper have also been discussed by Goicochea in Ref. [5]. The autor obtained self-consistent equations for $T_{\mathrm{c}}, \Delta(0)$, and used approximate temperature dependence of $\Delta$ near $T_{c}$. Here, we present explicit analytical expressions for these quantities as well as for the ratio $\Delta C / T_{\mathrm{c}}$. There is no essential difference between results derived in Ref. [5] and presented here. The analysis shows that the van Hove singularity in the conventional BCS theory cannot explain the large values of the order parameter $\Delta(T)$ near $T_{\mathrm{c}}$ and the specific heat jump [6].

Our starting point is the BCS gap equation

$$
1=V \int_{-\omega_{D}}^{\omega_{D}} \mathrm{~d} \varepsilon \frac{\rho(\varepsilon)}{2 \sqrt{\varepsilon^{2}+\Delta^{2}(T)}} \tanh \left(\frac{\sqrt{\varepsilon^{2}+\Delta^{2}(T)}}{2 k T}\right),
$$

where $V$ is the pairing potential and $\omega_{D}$ represents the characteristic phonon frequency. We consider a two-dimensional system where a logarithmic singularity in the density of states is situated at the Fermi level

$$
\rho(\varepsilon)=b_{1} \ln \left|\frac{\varepsilon}{b_{2}}\right| \text {. }
$$

In particular, for a two-dimensional square lattice and nearest-neighbor hopping integral $t$ the density of states is accurately reproduced with $b_{1}=-0.04687 t^{-1}$ and $b_{2}=21.17796 t[4]$.

Let us start with a discussion of the gap function at zero temperature, $\Delta(0)$. For $T=0$ the integral on the right-hand side of Eq. (1) can be exactly evaluated [7]. Then 


$$
\frac{2}{V b_{1}}=2 \ln \left(\frac{\Delta(0)}{b_{2}}\right) \ln \left(\frac{2 \omega_{D}}{\Delta(0)}\right)+\ln ^{2}\left(\frac{\omega_{D}}{\Delta(0)}\right)-\ln ^{2}(2)-\operatorname{Li}_{2}(1),
$$

where $\operatorname{Li}_{n}(z)$ is the polylogarithm function $\operatorname{Li}_{n}(z)=\sum_{k=1}^{\infty} z^{k} / k^{n}$ and $\operatorname{Li}_{2}(1)=$ $\pi^{2} / 6$. When carrying out the elementary algebraic transformations in (3), we obtain a simple expression for the gap function

$$
\Delta(0)=2 \omega_{D} \exp \left(-\frac{1}{\lambda_{1}}\right)
$$

where

$$
\frac{1}{\lambda_{1}}=\ln \left(\frac{\omega_{D}}{b_{2}}\right)+\left[\ln ^{2}\left(\frac{\omega_{D}}{b_{2}}\right)-\frac{2}{V b_{1}}-\frac{\pi^{2}}{6}\right]^{\frac{1}{2}} .
$$

On the other hand, one can adapt the formula for the superconducting transition temperature derived in Ref. [3]:

$$
k T_{\mathrm{c}}=a b_{2} \exp \left(-\frac{1}{\lambda_{2}}\right)
$$

where

$$
\frac{1}{\lambda_{2}}=\left[\ln ^{2}(2 a)+\ln ^{2}\left(\frac{\omega_{D}}{b_{2}}\right)-\frac{2}{V b_{1}}-2\right]^{\frac{1}{2}},
$$

and $a=2 \mathrm{e}^{\gamma} / \pi \approx 1.13$ ( $\gamma$ is the Euler constant). Then one, can easily obtain the ratio

$$
\frac{2 \Delta(0)}{k T_{c}}=\frac{4 \omega_{D}}{a b_{2}} \exp \left(\frac{1}{\lambda_{2}}-\frac{1}{\lambda_{1}}\right)
$$

Figure 1 shows the ratio of the gap parameter and the superconducting transition temperature as a function of the pairing potential. Here, we compare results calculated with the van Hove singularity with the value obtained within the standard BCS approach with a constant density of states $\left(2 \Delta(0) / k T_{\mathrm{C}} \approx 3.52\right)$. One can see that the logarithmic singularity in the density of states results in a slight enhancement of this quantity. The deviation from the standard BCS result is more visible for higher superconducting transition temperatures. However, the van Hove singularity itself cannot explain the experimental values of this ratio, which have been obtained for high-temperature superconductors [8].

In order to calculate the temperature dependence of the order parameter $\Delta(T)$ close to the transition temperature we follow the standard procedure discussed in Ref. [9]. With the help of the fermionic Matsubara frequencies, $\omega_{n}$, one can rewrite Eq. (1) in the form

$$
1=2 V k T \sum_{n} \int_{0}^{\omega_{D}} \mathrm{~d} \varepsilon \rho(\varepsilon) \frac{1}{\omega_{n}^{2}+\varepsilon^{2}+\Delta^{2}(T)} .
$$




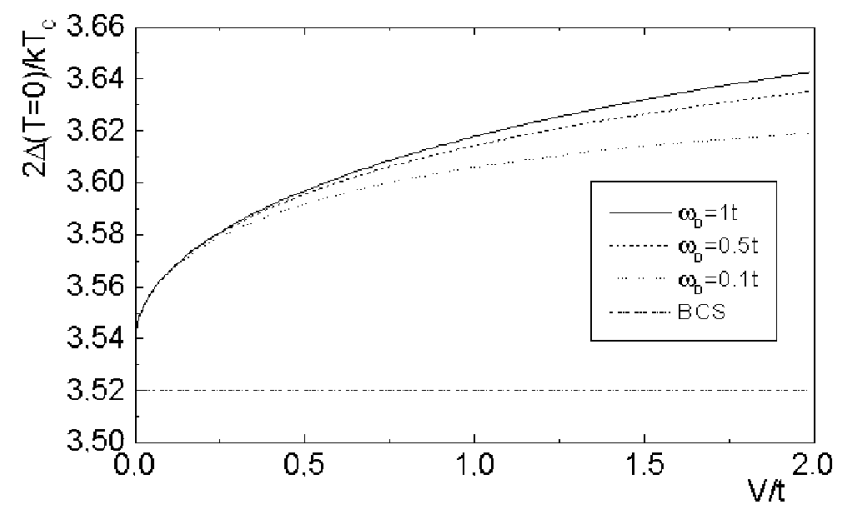

Fig. 1. The ratio $2 \Delta(0) / k T_{\mathrm{c}}$ as a function of the pairing potential $V$. The horizontal line indicates the value obtained within the standard BCS approach with a constant density of states.

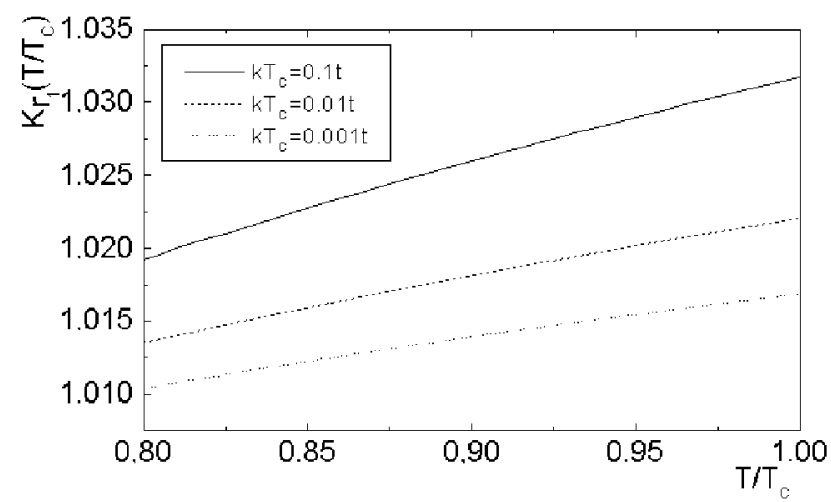

Fig. 2. $K r_{1}$ as a function of temperature calculated for different values of the superconducting transition temperature. See the text for details (Eq. (13)).

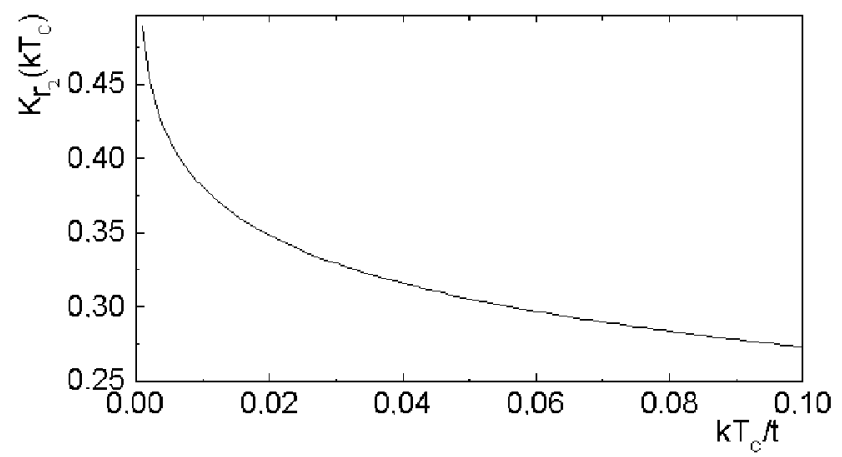

Fig. 3. $K r_{2}$ as a function of the transition temperature. See the text for details (Eq. (17)). 
The Taylor expansion around $T_{c}$ yields the following equations:

$$
\frac{1}{\omega_{n}^{2}+\varepsilon^{2}+\Delta^{2}(T)} \approx \frac{1}{\omega_{n}^{2}+\varepsilon^{2}}-\frac{\Delta^{2}(T)}{\left(\omega_{n}^{2}+\varepsilon^{2}\right)^{2}},
$$

which allows one to calculate the superconducting order parameter $\Delta(T)$

$$
\Delta^{2}(T) 2 k T \sum_{n} \int_{0}^{\omega_{D}} \mathrm{~d} \varepsilon \frac{\ln \left(\varepsilon / b_{2}\right)}{\left(\omega_{n}^{2}+\varepsilon^{2}\right)^{2}}=\left(1-\frac{T}{T_{\mathrm{c}}}\right)\left[\frac{T-T_{\mathrm{c}}}{2 T_{\mathrm{c}}}+\ln \left(\frac{k T_{\mathrm{c}}}{a b_{2}}\right)\right] .
$$

In the BCS regime $k T_{c} \ll \omega_{D}$. Therefore, when calculating the right-hand side of the above equation one can assume that $\omega_{D} \rightarrow \infty$. This simplification allows one to obtain an explicit expression for the gap function

$$
\Delta(T)=\pi k T\left(\frac{8}{7 \zeta(3)}\right)^{\frac{1}{2}}\left(1-\frac{T}{T_{c}}\right)^{\frac{1}{2}} K r_{1}(T)
$$

where

$$
K r_{1}(T)=\left[\frac{\ln \left(\frac{k T_{c}}{a b_{2}}\right)+\frac{T-T_{c}}{2 T_{c}}}{\ln \left(\frac{\pi k T}{b_{2}}\right)-\left[\frac{\mathrm{d}}{\mathrm{d} s} \ln \zeta(s)\right]_{s=3}-1.1}\right]^{\frac{1}{2}},
$$

and $\zeta$ denotes the Riemann zeta function. The difference between results obtained with a constant density of states [9] and the van Hove singularity shows up in the presence of $K r_{1}(T)$. Figure 2 shows this quantity as a function of temperature. One can see that $K r_{1} \approx 1$. Therefore, the presence of logarithmic singularity also hardly modifies the temperature dependence of the order parameter when compared to the standard BCS approach.

The specific heat jump that occurs at the transition temperature can be determined from the difference of thermodynamic potential between the superconducting and normal states [9]

$$
\Delta C=C_{\mathrm{S}}-C_{\mathrm{N}}=\left[-T\left(\frac{\partial^{2} \Delta \Omega}{\partial T^{2}}\right)_{N}\right]_{T_{\mathrm{c}}}
$$

where

$$
\Delta \Omega=\int_{0}^{\Delta} \mathrm{d} \Delta^{\prime}\left(\Delta^{\prime}\right)^{2} \frac{\mathrm{d}\left(\frac{1}{V}\right)}{\mathrm{d} \Delta^{\prime}}
$$

In the presence of the logarithmic singularity the integral, which enters the above equation, reads

$$
\Delta \Omega=-\frac{1}{2}(\pi k T)^{2}\left(\frac{8}{7 \zeta(3)}\right)\left(1-\frac{T}{T_{\mathrm{C}}}\right)^{2} K r_{2}(T)
$$

where

$$
K r_{2}(T)=b_{1} \frac{\left[\ln \left(\frac{k T_{c}}{a b_{2}}\right)+\frac{T-T_{c}}{2 T_{c}}\right]^{2}}{\ln \left(\frac{\pi k T}{b_{2}}\right)-\left[\frac{\mathrm{d}}{\mathrm{d} s} \ln \zeta(s)\right]_{s=3}-1.1} .
$$


Then, one can easily find an analytic expression for the jump of the specific heat

$$
\Delta C=K r_{2}\left(T_{\mathrm{c}}\right) \frac{8}{7 \zeta(3)}(\pi k)^{2} T_{\mathrm{c}} .
$$

Here, $K r_{2}$ represents a deviation from the linear dependence between $\Delta C$ and $T_{\mathrm{c}}$ which can be obtained with a constant density of states [9]. Figure 3 shows $\mathrm{Kr}_{2}$ as a function of the critical temperature. In the presence of the van Hove singularity the ratio $\Delta C / T_{\mathrm{c}}$ decreases with the enhancement of the critical temperature, whereas in the standard BCS formulation this ratio does not depend on the model parameters. Therefore, the van Hove singularity leads to a qualitative modification of the specific heat jump that occurs at the phase transition.

\section{Conclusions}

In the present work exact analytical formulae for the zero temperature energy gap, the ratio $2 \Delta(0) / k T_{c}$, temperature dependence of the order parameter near $T_{c}$, and the specific heat jump at the phase transition are derived with the van Hove singularity. Our results show that within a weak-coupling approach the ratio $2 \Delta(0) / k T_{c}$ hardly depends on the details of the density of states. In particular, the van Hove singularity leads only to a negligible enhancement of this quantity. The temperature dependence of the order parameter is also rather insensitive to the presence of logarithmic singularity in the density of states. However, this particular feature of the two-dimensional superconductors can show up in the jump of the specific heat, which takes place at the superconducting transition temperature. Here, one finds a qualitative difference with respect to the standard approach, which assumes a constant density of states. Namely, in the presence of the van Hove singularity the ratio of $(\Delta C) / T_{\mathrm{C}}$ decreases with an enhancement of the pairing correlations. This result suggests that two-dimensional features may be important when considering behavior of physical quantities at the superconducting phase transition. Note that this occurs on the BCS level. Within the strong-coupling theory the exact results are not yet accessible.

\section{Acknowledgments}

The authors are grateful for a useful discussion to Marcin Mierzejewski and Janusz Zieliński.

\section{References}

[1] J.G. Bednorz, K.A. Müller, Z. Phys. B 64, 189 (1986).

[2] D.J. Scalapino, Phys. Rep. 250, 329 (1995).

[3] R. Szczęśniak, M. Mierzejewski, J. Zieliński, P. Entel, Solid State Commun. 117, 369 (2001). 
[4] M. Maśka, M. Mierzejewski, Phys. Rev. B, in press.

[5] A.G. Goicochea, Phys. Rev. B 49, 6864 (1994).

[6] R.S. Markiewicz, J. Phys. Chem. Solids 58, 1179 (1997).

[7] A.P. Prudnikov, Yu. Brychkov, O.J. Marichev, Integrals and Series, Nauka, Moscow 1983.

[8] S. Ratanaburi, P. Udomsamuthirun, S. Yoksan, J. Supercond. 9, 485 (1996); J.M. Getino, M. de Liano, H. Rubio, Phys. Rev. B 48, 597 (1993).

[9] A.L. Fetter, J.D. Walecka, Quantum Theory of Many-Particle Systems, McGraw-Hill, 1971. 